\title{
Stathmin is Required for Stability of the Drosophila Neuromuscular Junction
}

\author{
Ethan R. Graf, ${ }^{1,3}$ Heather M. Heerssen, ${ }^{2}$ Christina M. Wright, ${ }^{1}$ Graeme W. Davis, ${ }^{2}$ and Aaron DiAntonio ${ }^{3}$ \\ ${ }^{1}$ Department of Biology and Neuroscience Program, Amherst College, Amherst, Massachusetts 01002, ${ }^{2}$ Department of Biochemistry and Biophysics, \\ Programs in Neuroscience and Cell Biology, University of California, San Francisco, San Francisco, California 94158, and ${ }^{3}$ Department of Developmental \\ Biology, Hope Center for Neurological Disorders, Washington University School of Medicine, St. Louis, Missouri 63110
}

Synaptic connections can be stably maintained for prolonged periods, yet can be rapidly disassembled during the developmental refinement of neural circuitry and following cytological insults that lead to neurodegeneration. To date, the molecular mechanisms that determine whether a synapse will persist versus being remodeled or eliminated remain poorly understood. Mutations in Drosophila stathmin were isolated in two independent genetic screens that sought mutations leading to impaired synapse stability at the Drosophila neuromuscular junction (NMJ). Here we demonstrate that Stathmin, a protein that associates with microtubules and can function as a point of signaling integration, is necessary to maintain the stability of the Drosophila NMJ. We show that Stathmin protein is widely distributed within motoneurons and that loss of Stathmin causes impaired NMJ growth and stability. In addition, we show that stathmin mutants display evidence of defective axonal transport, a common feature associated with neuronal degeneration and altered synapse stability. The disassembly of the NMJ in stathmin includes a predictable sequence of cytological events, suggesting that a common program of synapse disassembly is induced following the loss of Stathmin protein. These data define a required function for Stathmin during synapse maintenance in a model system in which there is only a single stathmin gene, enabling future genetic investigation of Stathmin function with potential relevance to the cause and progression of neuromuscular degenerative disease.

\section{Introduction}

The Drosophila neuromuscular junction (NMJ) has emerged as a model system for identifying genes and cellular processes that contribute to synapse maintenance (Eaton et al., 2002; Eaton and Davis, 2005; Pielage et al., 2005; Koch et al., 2008; Pielage et al., 2008; Massaro et al., 2009; Pielage et al., 2011). Studies of synapse retraction at the Drosophila NMJ have identified several proteins required for synapse stability, including the dynactin protein complex (Eaton et al., 2002), the presynaptic spectrin skeleton (Pielage et al., 2005), a giant isoform of Ankyrin2 (Koch et al., 2008; Pielage et al., 2008), and the actin-capping protein Adducin (Pielage et al., 2011). Here we describe Stathmin as an additional component of the machinery that is necessary to maintain NMJ stability.

Two large-scale genetic screens, each designed to identify mutations in genes that are necessary for NMJ stability, independently identified mutations in the stathmin gene. The assay

Received April 22, 2011; revised Aug. 25, 2011; accepted Aug. 29, 2011.

Author contributions: E.R.G., H.M.H., G.W.D., and A.D. designed research; E.R.G., H.M.H., and C.M.W. performed research; E.R.G., G.W.D., and A.D. wrote the paper.

This work was supported by NIH Grants NS047342 (G.W.D.), DA020812, NS065053, and NS043171 (A.D.) and an ALS Association grant (G.W.D.). We thank members of the DiAntonio and Davis labs for helpful discussions and Xiaolu Sun for technical assistance. We also thank André Sobel and Pernille Rørth for gifts of fly strains and antibodies, and the Bloomington Stock Center, the Vienna Drosophila RNAi Center, and the Developmental Studies Hybridoma Bank for fly strains and antibodies.

The authors declare no competing financial interests.

Correspondence should be addressed to Aaron DiAntonio, Department of Developmental Biology, Campus Box 8103, Washington University School of Medicine, St. Louis, M0 63110. E-mail: diantonio@wustl.edu.

DOI:10.1523/JNEUROSCI.2024-11.2011

Copyright $\odot 2011$ the authors $\quad 0270-6474 / 11 / 3115026-09 \$ 15.00 / 0$ employed in both screens is based on direct visualization of the larval NMJ in fixed tissue. Briefly, at the Drosophila larval NMJ, the presynaptic membrane is organized into a chain of boutons that are enveloped within the muscle cell (Collins and DiAntonio, 2007). The muscle membrane that surrounds individual synaptic boutons is composed of extensive muscle-membrane folds that create a postsynaptic structure termed the subsynaptic reticulum (SSR) (Budnik et al., 1996). The SSR can be labeled with antibodies directed against the scaffolding protein discs large (Dlg) (Budnik et al., 1996). The formation of the SSR and the clustering of postsynaptic proteins requires the innervation of the motoneuron terminal (Saitoe et al., 2001; Featherstone et al., 2002). Therefore, the presence of well-organized postsynaptic Dlg unapposed to a presynaptic terminal identifies places where the presynaptic terminal once resided and has since retracted. This conclusion is supported by numerous studies analyzing the NMJ at multiple stages of neuromuscular development using light-level, electron microscopy, and electrophysiological assays (Eaton et al., 2002; Eaton and Davis, 2005; Pielage et al., 2005; Koch et al., 2008; Pielage et al., 2008, 2011; Massaro et al., 2009). A similar assay is used routinely at the vertebrate neuromuscular junction as a measure of synapse elimination (Wernig et al., 1980).

Stathmin is a microtubule-binding protein that regulates microtubule dynamics and whose activity is modulated by phosphorylation, potentially linking diverse signaling systems to the control of microtubule stability (Belmont and Mitchison, 1996; Curmi et al., 1999; Gonatas et al., 2006; Tararuk et al., 2006; Watabe-Uchida et al., 2006; Westerlund et al., 2010). Stathmin has been associated with diverse models of neurodegeneration, 
axonal transport, nerve cell polarization, cancer, and the integrity of the Golgi complex (Curmi et al., 1999; Tararuk et al., 2006; Watabe-Uchida et al., 2006; Wen et al., 2010; Westerlund et al., 2010). However, the analysis of Stathmin in mouse knock-out studies has been complicated by the possibility of overlapping function among diverse Stathmin-like proteins that are encoded in the vertebrate genome (Schubart et al., 1996; Curmi et al., 1999). Here we present a genetic analysis of the stathmin gene at the Drosophila NMJ, a model system in which only a single stathmin gene is encoded (Ozon et al., 2002). Our data provide evidence that loss of Stathmin impairs axonal transport, attenuates neuromuscular growth, and destabilizes the neuromuscular junction such that the presynaptic nerve terminal retracts from the postsynaptic muscle cell. Our data are consistent with the observation of age-dependent neural degeneration in mice lacking Stathmin (Liedtke et al., 2002) and suggest a role for Stathmin in promoting NMJ stability.

\section{Materials and Methods}

Fly stocks. Flies were maintained at $25^{\circ} \mathrm{C}$ on standard fly food. Wild-type (WT) flies were Canton S (CS) or CS outcrossed to ELAV-GeneSwitch (Osterwalder et al., 2001) based on the experiment. The following fly lines were obtained from the Bloomington Stock Center: the P-element collection on the second chromosome (Bellen et al., 2004), the P-element line $\mathrm{PBac}\left\{5 \mathrm{HPw}^{+}\right\} \mathrm{stai}^{\mathrm{B} 200}$, the deficiency line $\mathrm{Df}(2 \mathrm{~L}) \mathrm{ED} 384$, and the line containing the repo-Gal4 driver (Sepp et al., 2001). The stathmin ${ }^{L 27}$ excision mutant and the lines containing the tub::stai, UAS-stai, and UASstai RNAi transgenes were obtained from P. Rørth (Institute of Molecular and Cell Biology, Singapore) (Borghese et al., 2006). The line containing the UAS-DLIMK1::HA transgene was described previously (Eaton and Davis, 2005). Neuronal expression of UAS-tagged transgenes was obtained using elav-Gal4 (Yao and White, 1994). The ELAVGeneSwitch driver was used to drive UAS-stai RNAi in neurons due to its ability to drive very strong expression. Muscle expression was obtained using the 24B-Gal4 driver (Brand and Perrimon, 1993). Muscle expression of UAS-gbb9.1 (Higashi-Kovtun et al., 2010) was obtained using the G7-Gal4 driver (Zhang et al., 2001).

Immunohistochemistry. Third-instar larvae of both sexes were dissected in PBS and fixed in either Bouin's fixative for 5 min or $4 \%$ formaldehyde for $20 \mathrm{~min}$. Larvae were washed with PBS containing $0.1 \%$ Triton X-100 (PBT) and blocked in 5\% NGS in PBT for 30 min, followed by overnight incubation in primary antibodies in 5\% NGS in PBT, three washes in PBT, incubation in secondary antibodies in 5\% NGS in PBT for $45 \mathrm{~min}$, three final washes in PBT, and equilibration in 70\% glycerol in PBS.

Samples were mounted in VectaShield (Vector Laboratories). The following primary antibodies were used: mouse $\alpha$-Brp (Bruchpilot) [1:250; mAb NC82; Developmental Studies Hybridoma Bank (DSHB)], mouse $\alpha$-Dlg (mAb 4f3; developed by C. S. Goodman, Renovis, San Francisco, CA), mouse $\alpha$-Futsch (1:1000; mAb 22C10; developed by S. Benzer, California Institute of Technology, Pasadena, CA), mouse a-acetylated tubulin (1:1000; Sigma), mouse $\alpha$-FasII $(1: 100 ; 1 D 4$; DSHB), rabbit $\alpha$-DVGLUT $(1: 10,000)$ (Daniels et al., 2008), rabbit $\alpha$-D-Stathmin- $\Delta$ C (1:1000; gift from A. Sobel, University Pierre et Marie Curie, Paris, France) (Ozon et al., 2002), rabbit $\alpha$-DGluRIII (1:2500) (Marrus et al., 2004), and rabbit $\alpha$-HA, 1:1000, (Santa Cruz Biotechnology). Goat Cy5and Cy3-conjugated secondary antibodies against mouse and rabbit IgG and Cy5-conjugated goat $\alpha$-HRP were used at 1:1000 and were obtained from Jackson ImmunoResearch. Antibodies obtained from the Developmental Studies Hybridoma Bank were developed under the auspices of the National Institute of Child Health and Human Development and maintained by the Department of Biological Sciences of the University of Iowa (Iowa City, IA).

RU486-GeneSwitch. The RU486-GeneSwitch system was administered according to Osterwalder et al. (2001). For expression of UAS-stai RNAi driven by ELAV-GeneSwitch, females were maintained on fly food con- taining $25 \mathrm{mg} / \mathrm{ml}$ RU486 (mifepristone; Sigma) for $2 \mathrm{~d}$ before mating and allowed to lay eggs on RU486-containing fly food.

Western blots. Third-instar larval brains were homogenized in ice-cold homogenization buffer (67m $\mathrm{m}$ Tris- $\mathrm{HCl}, \mathrm{pH} 8.0 ; 67 \mathrm{~mm} \mathrm{NaCl} ; 2 \mathrm{M}$ urea, 1 mM EDTA, and 1.3\% SDS), and samples were run on $12 \%$ SDS-PAGE gels according to standard procedures. Rabbit $\alpha$-D-Stathmin- $\Delta \mathrm{C}$ (gift from A. Sobel, University Pierre et Marie Curie, Paris, France) (Ozon et al., 2002) was used at 1:2000. Mouse- $\alpha$-actin (JLA20; DSHB) was used at 1:50. HRP-conjugated goat $\alpha$-rabbit and $\alpha$-mouse (Jackson ImmunoResearch) were used at 1:10,000.

Imaging and analysis. Samples were imaged using a Nikon C1 confocal microscope. All genotypes for an individual experiment were imaged at the same gain and set such that signals from the brightest genotype for a given experiment were not saturating. Images for quantifying the average Futsch intensity per NMJ and average Stathmin and Brp intensity per nerve segment were randomized and analyzed using MetaMorph software (Universal Imaging). To determine the area of the NMJ or nerve within which to measure Futsch or Brp, respectively, NMJ outlines were delineated by HRP and the presynaptic vesicle protein vesicular glutamate transporter (DVGLUT) signal, and the nerve area was delineated by HRP signal. The percentage of NMJs with footprints and bouton number per NMJ were counted manually. NMJs with at least one obvious bouton-like patch of Dlg that was unopposed to DVGLUT were identified as synaptic retraction events. For bouton number quantification, only NMJs with no obvious retraction events were included in the quantification, and individual boutons were identified as discrete patches of DVGLUT. Statistical analysis was performed using ANOVA for comparison of samples within an experimental group. All histograms and measurements are shown as mean \pm SEM.

\section{Results}

Mutations in the stathmin gene were identified in two independently designed and executed genetic screens. In each screen, third-instar homozygous mutant larvae containing insertions of transposable elements were costained for presynaptic and postsynaptic markers, either DVGLUT with the postsynaptic SSR protein Dlg or the presynaptic marker Brp with the essential glutamate receptor subunit DGluRIII. Immunostained NMJs were screened for the presence of postsynaptic footprints in which bouton-like patches of Dlg or DGluRIII are unopposed by presynaptic markers, a hallmark of synapse retraction (Eaton et al., 2002). In total, an overlapping set of $>3500$ mutant lines were screened by direct visualization of the NMJ in abdominal segments A2-A6. Mutations in the stathmin gene were identified as having pronounced synapse retraction (Fig. $1 A$ ). In a stathmin mutant, Dlg patches in the muscle that are no longer apposed to axonal boutons containing DVGLUT can be readily discerned (arrowheads). A phenotype similar is observed when immunostaining for the active zone protein Brp as the presynaptic component and DGluRIII as the postsynaptic component (data not shown). The severity and prominence of the retraction phenotype increases in an anterior-to-posterior manner, becoming most acute in posterior segments. In the strongest allele identified, the percentage of muscle 4 NMJs with postsynaptic footprints is significantly greater in segment A5 as compared to segment A3 (A5, $77 \pm 8 \%$; A3, $12 \pm 6 \% ; n=26$ NMJs each; $p \ll$ $0.001)$. However, despite NMJ retraction, these mutants are viable and fertile.

\section{stathmin mutants exhibit defective synaptic stability}

A total of four independent transposon insertion mutations were found to share the stathmin mutant phenotype. We chose to focus in detail on a single transposable element, $\mathrm{PBac}\left\{5 \mathrm{HPw}^{+}\right\}$ $s t a i^{B 200}$ (referred to as $s t a i^{B 200}$ ), that is situated within an intron of the coding sequence of the stathmin (stai) gene. We also 
identified a deficiency chromosome, $D f(2 L) E D 384$, that uncovers the stai gene. When $s t a i^{B 20 O}$ is placed in trans to this deficiency, we find large numbers of synapse retractions that are similar in size and frequency to that observed in $\operatorname{stai}^{B 200}$ alone. In addition, analysis of a small deletion, stathmin $^{\text {L27, }}$, which uncovers the entire stathmin gene as well as four adjacent genes (Borghese et al., 2006), also fails to complement the stai ${ }^{B 200}$ mutation. The retraction phenotype of the $s t a i^{B 200}$ homozygotes and transheterozygotes of stai $^{B 200}$ and stathmin ${ }^{L 27}$ are indistinguishable. The percentages of muscle 4 NMJs in segment A4 that have postsynaptic footprints are similar between the two genotypes $\left(\right.$ stai $^{B 200}, 69 \pm 9 \%, n=26$ stai $^{B 200}$ / stathmin $\left.^{L 27}, 71 \pm 9 \%, n=24 ; p>0.9\right)$, and the sizes of those retractions are also similar $\left(\right.$ stai $^{B 200}, 3.9 \pm 0.8$ boutons/retraction; stai ${ }^{B 200} /$ stathmin $^{L 27}, 3.7 \pm 0.4$ boutons/retraction; $p>0.7 ; n=17$ NMJs per genotype at muscle 4 , segment A4). Thus, stai $^{B 20 O}$ behaves as a strong loss-offunction or genetic null mutation for the synaptic retraction phenotype. Based on these data, we conclude that the phenotype of synapse retraction maps to the stathmin locus, suggesting that loss of stathmin causes synapse disassembly and retraction at the NMJ.

We also quantified postsynaptic footprints at muscles $6 / 7$ as performed in previous studies of synapse retraction in this system (Eaton et al., 2002; Massaro et al., 2009; Pielage et al., 2011). The percentage of synapses at muscles $6 / 7$ showing a synaptic footprint was significantly increased compared to wild type, both when analyzing only segment 4 (wild type, $4 \% \pm 4 ;$ stai $^{B 200}, 80 \% \pm 8 ; n=26 ; p$ $\ll 0.001$ ) and when averaging data from segments A3-A5 (wild type, $1 \% \pm 1, n=71 ;$ stai $\left.^{B 200}, 69 \% \pm 5, n=78 ; p \ll 0.001\right)$. These data demonstrate that stathmin mutations affect synapse stability at multiple muscles in the Drosophila neuromuscular system.

Next, we used an antibody to Drosophila Stathmin to assess whether $s t a i^{B 20 O}$ disrupts Stathmin protein expression. We show that the Stathmin antibody recognizes bands of the predicted size on immunoblots from wild-type larvae and that there is much less protein expressed in the $s t a i^{B 20 O}$ mutant compared to controls (Fig. $1 B$ ). While some Stathmin protein is still expressed in homozygous mutant larvae, protein levels are further reduced in immunoblots from $s t a i^{B 20 O} / D f(2 L) E D 384$ larvae, indicating that $\operatorname{stai}^{B 200}$ is not a protein null mutation, though it behaves genetically as a strong loss-of-function mutation (see above).

To confirm that synapse retraction at the NMJ in $s t a i^{B 200}$ is due to loss of Stathmin, we transgenically expressed stathmin in the $s t a i^{B 20 O}$ background. Transgenic expression of stathmin from the ubiquitous tubulin promoter (tub::stai) (Borghese et al., 2006) rescues the $s t a i^{B 200}$ mutant axonal retraction phenotype (Fig. $1 A, C$ ). Whereas axonal retractions and their resulting postsynaptic footprints are very rarely observed in wild-type larvae, $\sim 70 \%$ of homozygous stai ${ }^{B 20 O}$ muscle 4 NMJs in segment 4 are in the process of retraction (Fig. 1C). Transgenic stathmin expres- sion reduces the occurrence of observed retractions to $\sim 10 \%$. Based on these data, we conclude that the retraction phenotype in the $s t a i^{B 200}$ mutant is due to the loss of stathmin and, by extension, that stathmin is required for NMJ stability.

We also quantified synaptic bouton numbers in the stathmin mutant. In an attempt to separate the phenotypes of synaptic retraction and synaptic growth, bouton numbers were quantified at NMJs without any histological evidence of synaptic footprints. When this selection is done, the $s t a i^{B 20 O}$ mutant NMJs are still small and have fewer boutons than controls. For example, NMJs at muscle 4 in the stai $^{B 200}$ mutant have $40 \%$ fewer boutons than wild type (Fig. 1D). This reduction in bouton number is fully rescued by the transgenic expression of $t u b:: s t a i$. This suggests that Stathmin may have a role in neuromuscular growth. However, we cannot rule out the possibility that synapse retractions occurred at these NMJ at earlier stages of neuromuscular development, causing the decrease in total bouton number at the stathmin mutant NMJ, even though there is no evidence of ongoing retraction.

\section{Disruption of neuronal stathmin results in synaptic defects}

The synaptic phenotype observed in the stathmin mutants suggests a role for Stathmin in neurons. Immunostaining wild-type larvae with anti-Stathmin reveals prominent staining in motor nerves (Fig. 2A). Costaining with the neuronal membrane marker HRP allows for the recognition of two separate compartments within the nerve: (1) an HRP-positive inner region that contains both bundles of axons as they stream through the nerve and the inner ensheathing glial cells that wrap around individual axons, and (2) the outer perineurial glial cells that wrap their 


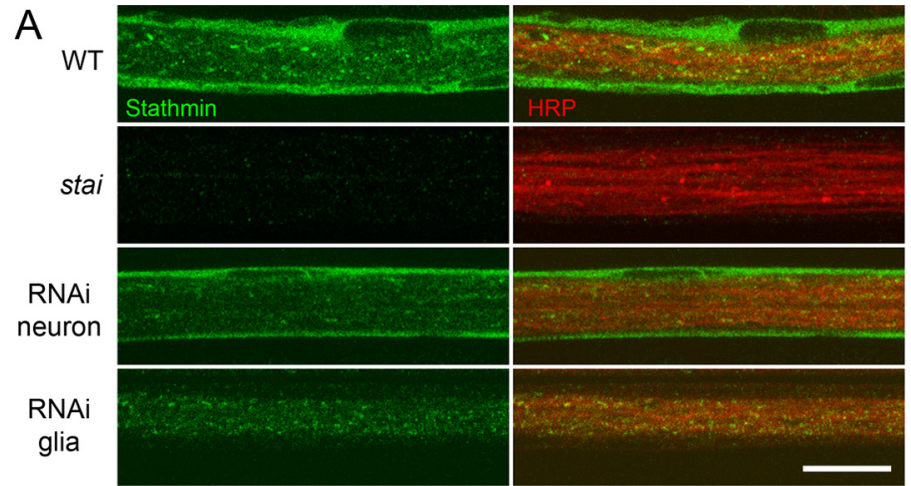

B

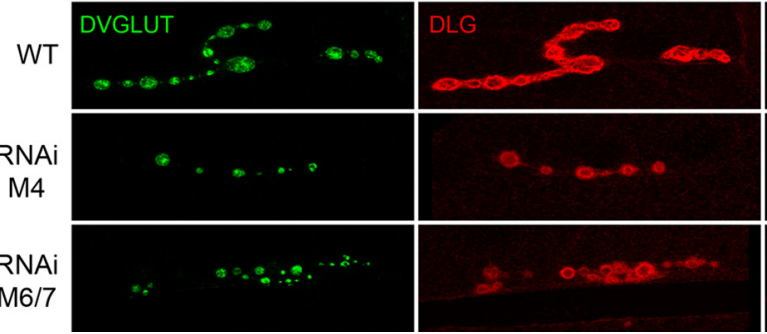

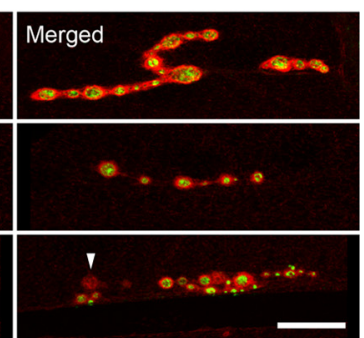

Figure 2. Neuronal expression of stathmin RNAi reduces NMJ size and results in axon retraction. $\boldsymbol{A}$, Images of single optica sections of motor nerves immunostained with $\alpha$-Stathmin (green) and $\alpha$-HRP (red). Immunostaining of WT nerves reveals Stathmin staining in both the outer perineurial glial cells that surround the HRP-positive region and within the HRP-positive region itself, which contains axons and inner ensheathing glial cells. In contrast, Stathmin signal is significantly decreased in the stathmin mutant $\left(\right.$ stai $^{B 200} /$ stai $^{\text {B200}}$ ). Neuronal expression of stathmin RNAi (UAS-stai RNAi/ $+;+/+;$ GeneSwitchELAV/+) reduces Stathmin signal in the HRP-positive region of the axons. Conversely, glial expression of stathmin RNAi (UAS-stai RNAil $+;+/+$; repo-Gal4/+) decreases Stathmin signal in the glial cells and allows clearer visualization of axonal Stathmin. $\boldsymbol{B}$, Images of segment 4 NMJs from WT (GeneSwitchELAV/+) and following neuronal expression of stathmin RNAi (UAS-staiRNAi/ + ; + + ; GeneSwitchELAV/+). Immunostaining for $\alpha$-DVGLUT (green) and $\alpha$-Dlg (red) reveals a reduction in muscle 4 NMJ size and bouton number following neuronal expression of stathmin RNAi (middle row) compared to WT (top row). Axon retractions are also detected at muscle 6/7 NMJs following stathmin RNAi expression (bottom row) by the presence of postsynaptic Dlg footprints (arrowhead). Scale bars: $10 \mu \mathrm{m}$.

processes around the inner glial cells and axons (Banerjee et al., 2006). Analyzing single optical sections that bisect the nerve lengthwise shows stathmin expression both in the outer perineurial glia cells and in the HRP-positive region containing the axons and inner ensheathing glial cells. Stathmin staining is substantially reduced in both the glial and axonal regions of peripheral nerves in homozygous stai ${ }^{B 200}$ mutant larvae (Fig. $2 A$ ). Tissue-specific expression of a UAS-RNAi transgene targeting the stathmin transcript (Borghese et al., 2006) by the UAS/Gal4 expression system allows clearer visualization of neuronal versus glial expression. Neuron-specific expression of stathmin RNAi via the strong neuronal driver GeneSwitchELAV decreases the average intensity of Stathmin in the region associated with HRPpositive axons (Fig. $2 \mathrm{~A}$; wild type, $83 \pm 9$ a.u.; stathmin $\mathrm{RNAi}$, $42 \pm 4$ a.u.; $p<0.001 ; n=12$ nerve segments each) but has no significant affect on average Stathmin intensity in the outer perineurial glia (wild type, 100 a.u. \pm 6 ; stathmin RNAi, 92 a.u. \pm $8 ; p>0.4 ; n=12$ nerve segments each). Conversely, glial-specific expression of stathmin RNAi via the pan-glial driver repo-Gal4 significantly reduces the average intensity of Stathmin in the outer perineurial glial region surrounding the HRP-positive axonal region (Fig. $2 A$; wild type, $100 \pm 10$ a.u.; stathmin RNAi, $31 \pm 1$ a.u.; $p \gg 0.001 ; n=11$ nerve segments each). Glial expression of stathmin RNAi also moderately reduces average Stathmin intensity in the HRP-positive axonal region (wild type, $72 \pm 4$ a.u.; stathmin RNAi, $56 \pm 2$ a.u.; $p<0.005 ; n=11$ nerve segments each). Since this region contains the inner ensheathing glial cells that wrap their processes directly around the axons in addition to the axons themselves, decreased stathmin expression likely reflects the loss of Stathmin in the inner glial cells, although we cannot exclude the possibility that there may be leakage of the repo-Gal4 driver resulting in mild knockdown in neurons as well. Regardless, the dramatic loss of Stathmin from glia allows for the clear visualization of axonal Stathmin (Fig. $2 A$, bottom panels). While stathmin is clearly present in the axon, stathmin staining is difficult to detect in the motor neuron nerve terminal at the NMJ due to high background staining in muscles that overwhelms the Stathmin signal at the NMJ.

Stathmin is expressed in multiple cell types and so could be required in any of these tissues for synapse stability. To address this issue, we selectively knocked down stathmin in glia, neurons, and muscle. Even though Stathmin is prominently expressed in the peripheral glia surrounding motoneuron axons, expression of stathmin RNAi by the pan-glial repo-Gal4 driver does not reduce bouton number at muscle 4 NMJs (wild type, $21.4 \pm 1.3$ boutons per NMJ; stathmin RNAi, $23.0 \pm 1.0$ boutons per NMJ, $n=22 \mathrm{NMJ}$ each; $p>$ 0.3 ) and results in no observable footprints at either muscle 4 or muscle $6 / 7$ NMJs. In contrast, neuron-specific expression of stathmin RNAi via the GeneSwitchELAV driver results in an NMJ phenotype similar to but slightly weaker than that observed in the $s t a i^{B 200}$ mutant (Fig. 2B). Muscle 4 NMJs expressing stathmin RNAi are significantly smaller than control NMJs with a $43 \%$ reduction in bouton number at NMJs that do not show evidence of synaptic footprints (Fig. $2 \mathrm{~B}$; wild type, $20.3 \pm 0.6$ boutons per NMJ, $n=26 \mathrm{NMJ}$; stathmin RNAi, $11.6 \pm 0.9$ boutons per NMJ, $n=29$ NMJ; $\mathrm{p} \ll 0.001)$. In addition, synaptic retractions are observed in $\sim 40 \%$ of muscle $6 / 7$ NMJs in segment 4 (Fig. 2 B; $43.3 \pm 9 \%$ of NMJs showed synapse retractions, $\mathrm{p} \ll 0.001$ compared to wild type). A similar synaptic retraction phenotype was observed when using two additional UAS-stathmin RNAi transgenes driven in the nervous system that targeted independent sequences in the stathmin transcript, arguing for the specificity of this effect (RNAi2: $23.6 \pm 5 \%$ of NMJs showed synapse retractions, $p<0.01$ compared to wild type; RNAi3: $20.3 \pm$ $6 \%$ showed synapse retractions, $p<0.01$ compared to wild type: note that in this analysis the percentage of synapses with footprints was averaged across segments A2-A5). Furthermore, expression of stathmin RNAi by the muscle-specific 24B-Gal4 driver fails to reduce bouton number at muscle 4 NMJs (wild type, $21.7 \pm 1.2$ boutons per NMJ, $n=14$ NMJs; stathmin RNAi, $25.5 \pm 1.0$ boutons per NMJ, $n=12$ NMJs; $p<0.05)$ and does not result in synaptic retraction at muscle 4 or $6 / 7$ NMJs. The ability of neuron-specific but not glial- or muscle-specific Stathmin knockdown to phenocopy the stai $^{B 200}$ mutant phenotype indicates that Stathmin acts in the neuron to prevent NMJ retraction. 

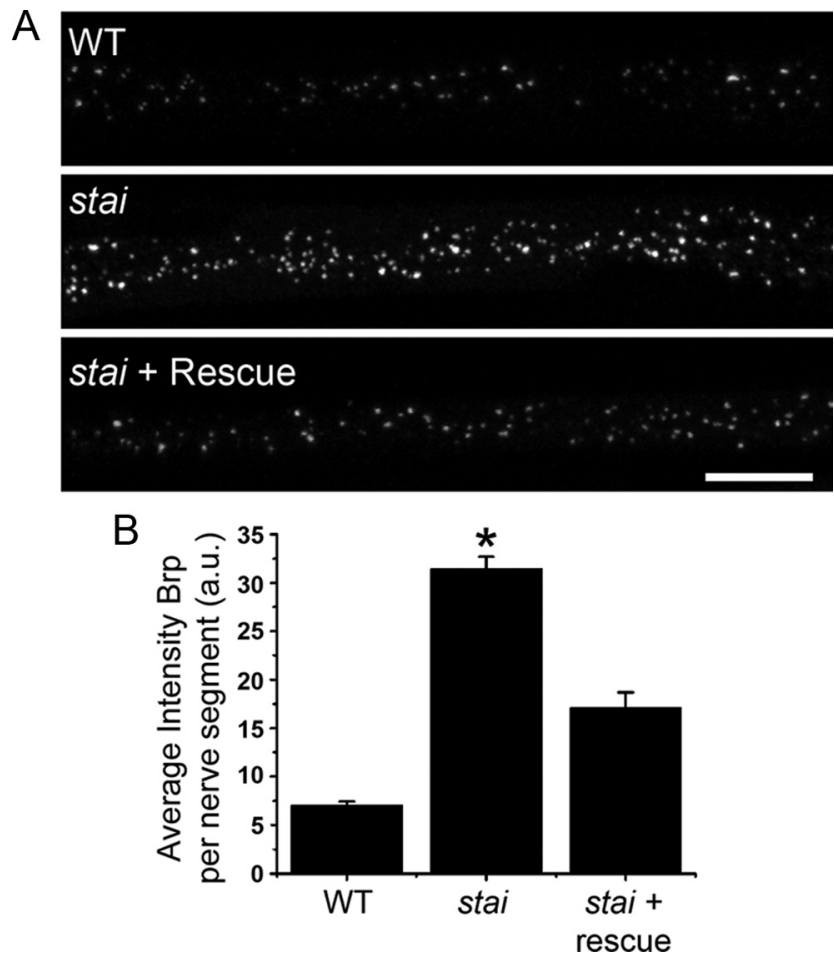

Figure 3. Brp accumulates in stathmin mutant nerves. $\boldsymbol{A}$, Images of $\alpha$-Brp immunostaining in nerve segments from WT $(+/+)$, the stathmin mutant $\left(\right.$ stai $^{B 200} /$ stai $\left.^{B 200}\right)$, and the stathmin mutant with rescue expression of tub::stai (stai ${ }^{8200} /$ stai $^{\text {B200; }}$ tub::stai/tub::stai). Scale bar, 10 $\mu \mathrm{m} . \boldsymbol{B}$, Histogram showing the quantification of the average intensity of Brp per nerve segment in the three genotypes. WT, $n=14$; stathmin mutant $n=14$; stathmin mutant with rescue, $n=13 .{ }^{*} p \ll 0.001$ versus wild type and rescue.

While neuron-specific knockdown of Stathmin results in stunted synaptic growth and increases retraction events, overexpression of UAS-stathmin (Borghese et al., 2006) in wild-type motoneurons does not induce synapse retraction $(1.5 \pm 1.5 \%$ of NMJs show evidence of retraction in elav-Gal4; UAS-stathmin animals; $p>0.3$ compared to wild type). Rather, UAS-stathmin overexpression leads to increased levels of Stathmin in the synaptic terminal and causes a mild increase in neuronal branching without an associated change in synaptic bouton number (data not shown).

\section{stathmin is required for axon transport}

It was demonstrated previously, both in Drosophila (Eaton et al., 2002) and mice (LaMonte et al., 2002; Hafezparast et al., 2003), that loss of genes necessary for normal axonal transport can cause neuromuscular synapse retraction and degeneration. Because Stathmin regulates microtubule dynamics and integrity in other systems, we sought to determine whether axonal transport is altered in stathmin mutants. The formation of axonal protein aggregates composed of synaptic material is considered a hallmark of impaired axonal transport (Hurd and Saxton, 1996). Therefore, we analyzed the levels of the active zone protein Brp in peripheral nerves as they extend between the ventral nerve cord and the periphery. Brp is readily observed in wild-type nerves as it is transported down axons. In $s t a i^{B 200}$ mutant nerves, we found increased levels of axonal Brp. The average intensity of Brp per nerve segment is five times greater than in wild types (Fig. 3), indicating a substantial accumulation of Brp within the axon. Transgenic expression of $t u b:: s t a i$ in the $s t a i^{B 200}$ mutant significantly reduces the accumulation of Brp in nerves, indicating that the defect in axonal transport is caused by loss of Stathmin. Similar results are observed for a second axonal cargo, the vesicular glutamate transporter (data not shown), indicating that this is a general defect and not specific to the transport of the Brp protein. In addition, knockdown of stathmin via the transgenic expression of an RNAi construct in neurons results in a similar axon transport phenotype (data not shown). While this aberrant accumulation of synaptic proteins in the axon is consistent with a defect in axonal transport, we cannot rule out the possibility that it is a secondary consequence of NMJ retraction and degeneration. However, this accumulation still occurs in segmental nerves to anterior segments, where NMJ retraction is rare. Hence, we prefer the interpretation that this phenotype reflects defects in axonal transport.

To determine whether stathmin disruption affects axonal microtubules and their associated proteins, we immunostained the $s t a i^{B 20 O}$ mutant for Futsch, a neuron-specific microtubuleassociated protein that is a marker of the neuronal microtubule cytoskeleton (Hummel et al., 2000). In wild-type neurons, Futsch staining is prominent along the entire axon, including the axon terminal where it extends down the length of the NMJ (Roos et al., 2000). In the $s t a i^{B 200}$ mutant, the average Futsch intensity in peripheral nerves is the same as in wild type (WT, $73 \pm 4$ a.u., $n=$ 16; stai ${ }^{B 200}, 77 \pm 5$ a.u., $\left.n=17 ; p>0.6\right)$. There is no obvious alteration in the apparent organization of the Futsch staining within the axons, suggesting that the observed defects in axonal transport may not be secondary to changes in the underlying microtubule cytoskeleton.

The $s t a i^{B 200}$ mutant NMJ does, however, show decreased levels of Futsch within the presynaptic terminal that is partially rescued by transgenic expression of tub::stai (Fig. 4A,C). A similar loss of Futsch is observed following neuronal expression of stathmin RNAi (Fig. 4B,C). This decrease in Futsch is selective for the axon terminal since normal Futsch staining is observed in axon shafts directly upstream of NMJs. It should be noted that whereas axon retractions are most often observed in posterior segments of stai $^{\mathrm{B} 200}$ mutants, the loss of synaptic Futsch staining is prominent within every segment. There is a clear reduction in Futsch intensity even in the most anterior segments, where other synaptic markers such as DVGLUT appear normal. Since Futsch is a microtubule-associated protein, its deficiency does not directly indicate a loss of synaptic microtubules within the presynaptic nerve terminal. However, given that Futsch functions to stabilize synaptic microtubules, its loss suggests that the integrity of the synaptic microtubule cytoskeleton is compromised in $s t a i^{\mathrm{B} 200}$ mutants. To provide further evidence in support of this conclusion, we stained the NMJ for acetylated tubulin. In wild-type NMJs, acetylated tubulin extends down the entire axon terminal (Roos et al., 2000) (Figure 5A). How-

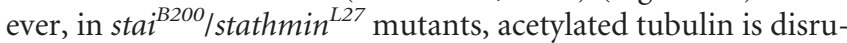
pted in a manner similar to Futsch (Fig. 5B). The disappearance of acetylated tubulin and Futsch is consistent with a model in which the loss of synaptic microtubules and microtubuleassociated proteins occurs during the earliest phases of synapse destabilization and retraction.

\section{Axon terminal retraction occurs in sequential stages}

The presence of DVGLUT in the absence of Futsch at retracting nerve terminals is consistent with previous observations that retraction of the presynaptic terminal includes the sequential loss of different synaptic antigens (Pielage et al., 2008). For example, it was demonstrated previously that loss of Futsch-positive microtubules precedes the loss of cytoplasmic synaptic markers during 

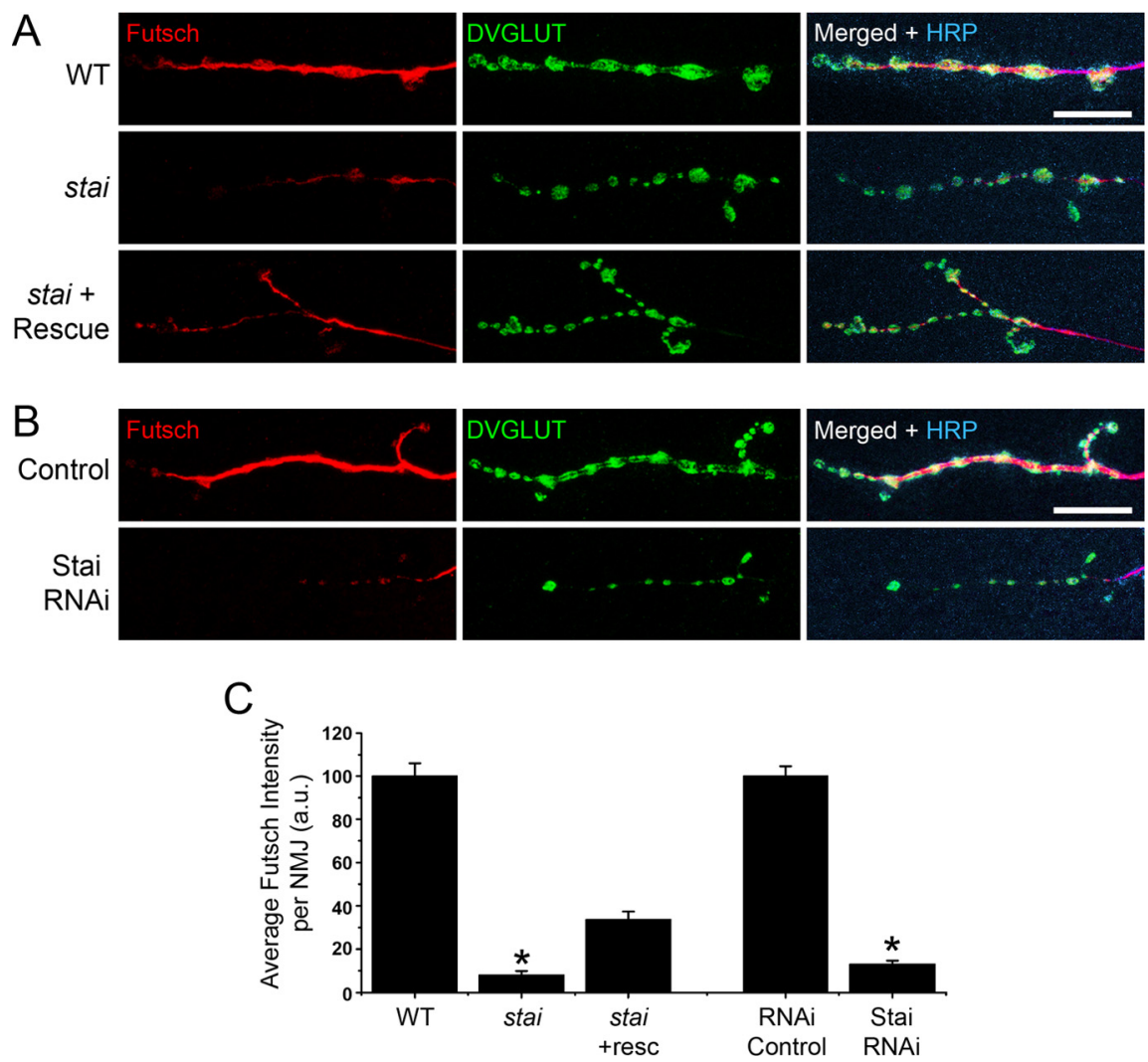

Figure 4. stathmin disruption results in loss of Futsch at the axon terminal. $A$, Images of muscle 4 NMJs in segment 4 from WT

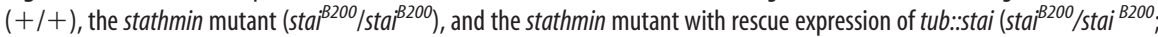
tub::stailtub::stai) immunostained with $\alpha$-Futsch (red), $\alpha$-DVGLUT (green), and $\alpha$-HRP (blue). $\boldsymbol{B}$, Images of muscle 4 NMJs in segment 4 from WT (GeneSwitchELAV/+) and following neuronal expression of stathmin RNAi (UAS-stai RNAi/+; +/+; GeneSwitchELAV/+) immunostained with $\alpha$-Futsch (red), $\alpha$-DVGLUT (green), and $\alpha$-HRP (blue). C, Histogram showing the quantification of the average intensity of Futsch NMJ in the genotypes shown in $A$. WT, $n=11$; stathmin mutant, $n=10$; stathmin mutant with rescue, $n=13$; RNAi control, $n=14$; stathmin RNAi, $n=10$. ${ }^{*} p<0.001$ for the comparisons wild type versus rescue and RNAi control versus stathmin RNAi. Scale bars: $10 \mu \mathrm{m}$.

synapse retraction. The loss of cytoplasmic proteins is followed by the disorganization and loss of cell adhesion molecules. Finally, there is a prominent fragmentation of the synaptic plasma membrane (Pielage et al., 2008). To better understand NMJ retraction in the $s t a i^{B 20 O}$ mutant, we performed a similar analysis by immunostaining stai $^{B 20 O} /$ stathmin $^{L 27}$ mutant NMJs with various synaptic markers.

The cytoplasmic serine/threonine kinase LIM kinase1 (LIMK1) is involved in the synapse-stabilizing activity of the BMP receptor (Eaton and Davis, 2005). Therefore, we asked whether its synaptic localization is also disrupted in stathmin mutant NMJs as a precursor to retraction. Neuronally driving UAS-DLIMK1::HA with elav-Gal4 in a wild-type background results in the localization of DLIMK1 throughout the nerve terminal (Fig. 5A). Transgenic DLIMK1::HA has a similar localization pattern in the $s t a i^{B 200} /$ stathmin ${ }^{L 27}$ mutant, and is present in both retracting and nonretracting NMJs that are devoid of Futsch staining (Fig. 5B). DLIMK1::HA is lost from retracting NMJs along with other active-zone and vesicle-associated proteins including Brp and DVGLUT (Fig. 5B). In most retracting axon terminals, distal patches of the axonal membrane marker HRP are devoid of DLIMK1, Brp, and DVGLUT, consistent with the eventual disassembly of the synaptic plasma membrane observed previously at the light (Pielage et al., 2008) and EM levels (Eaton et al., 2002; Pielage et al., 2011). The terminal patches of HRP are indicative of "nerve terminal remnants" and are positive for the cell adhesion molecule FasII (Fig. 5B). Together, these data are consistent with previously proposed models of synapse retraction, indicating that the loss of Stathmin may induce a synapse disassembly program that is common to different mutations.

It is worth noting that two of the mutations for which such an analysis of synapse retraction has been performed are microtubule-associated (Stathmin) or microtubule-interacting (Ankyrin2-L) proteins. As such, it is formally possible that this particular disassembly program is specific to cellular perturbations that directly affect the stability of the microtubule cytoskeleton. However, genetic mutations in the futsch gene, which cause the fragmentation of synaptic microtubules, do not induce subsequent NMJ retraction (Roos et al., 2000; Pielage et al., 2008). Therefore, it seems unlikely that disruption of synaptic microtubules is the cause of NMJ retraction in the stathmin mutant. Ultimately, the precise cellular perturbation that initiates the proposed synapse disassembly program remains unknown (Goda and Davis, 2003).

\section{Discussion}

Here we show that Stathmin is required for the maintenance of the Drosophila neuromuscular junction. In a stathmin mutant, motoneuron terminals retract from previously innervated muscles. The severity of impaired synapse stability follows an anterior-to-posterior gradient, with denervation being more commonly observed in posterior segments. We also demonstrate that NMJs that are not in the process of retraction have significantly fewer synaptic boutons than wild type, suggesting that Stathmin may also be necessary for normal synaptic growth. In addition, the stathmin mutant exhibits hallmarks of a defect in axonal transport, including an accumulation of synaptic proteins within peripheral nerves.

Several studies have reported alterations in neurite growth and stability following altered expression of stathmin family members (Grenningloh et al., 2004) that are consistent with a function of Stathmin for synaptic growth and stability observed at the Drosophila NMJ. Overexpression of SCG10 in PC12 cells increases neurite growth in response to NGF (Riederer et al., 1997), whereas reduction of SCG10 expression in rat primary hippocampal cultured neurons via RNAi results in a reduction in neurite elongation rate (Morii et al., 2006). Interestingly, stathmin knock-out mice show no neurodevelopmental phenotype and display no neurological or behavioral deficits as young adults (Schubart et al., 1996). The lack of developmental defects in the stathmin knock-out mouse may be due to compensation by the other stathmin family members. However, aged stathmin mutant mice develop a late-onset axonopathy of the peripheral nervous system and CNS (Liedtke et al., 2002), a phenotype highly reminiscent of 
the numerous retracting axon terminals observed in the Drosophila stai ${ }^{B 200}$ mutant.

\section{Neuronal Stathmin activity and NMJ retraction}

Stathmin is a tubulin-binding cytosolic phosphoprotein that is one member of a family of related proteins in vertebrates that also includes SCG10, SCLIP, and RB3 (Curmi et al., 1999). A single stathmin gene has been identified in Drosophila and displays similar biochemical and functional characteristics as its vertebrate relatives (Ozon et al., 2002). Stathmin family members are expressed ubiquitously in multiple cell types in vertebrates (Curmi et al., 1999). In Drosophila, expression of stathmin has been studied primarily in the embryo, focusing on the embryonic nervous system, pole cells, and border cells (Ozon et al., 2002; Borghese et al., 2006). Consistent with stathmin mRNA expression in embryonic neurons, we show immunostaining of Stathmin in the axons of third-instar motoneurons. Thus, stathmin is expressed at the correct time and place to influence motoneuron development and stability.

To date, studies of Stathmin have focused on its role as a potent destabilizer of microtubules, balancing the effects of microtubule-associated proteins that act to stabilize microtubules by promoting their assembly and cross-linking them to form bundles (Grenningloh et al., 2004). Numerous studies have attempted to elucidate the mechanism by which Stathmin induces its microtubule-destabilizing activity. In a phosphorylation-regulated manner, Stathmin binds to and sequesters free tubulin dimers, decreasing the concentration of tubulin subunits available for incorporation into growing microtubules and maintaining a tubulin pool (Walker et al., 1988; Belmont and Mitchison, 1996; Curmi et al., 1997; Jourdain et al., 1997; Lachkar et al., 2010). Thus, Stathmin may increase catastrophe frequency and/or slow microtubule growth rate by lowering the availability of tubulin for polymerization. Alternatively, Stathmin may directly interact with microtubules to promote depolymerization independent of tubulin sequestration by altering the GTP state of microtubules (Howell et al., 1999).

Our data demonstrate that the loss of Stathmin causes synapse retraction and an associated loss of stabilized microtubules within the presynaptic nerve terminal. Since Stathmin destabilizes microtubules, stathmin disruptions would be expected to enhance microtubule stability rather than result in a loss of microtubules. However, since dynamic instability of microtubules is important for normal function, enhanced stability of certain populations of microtubules combined with a reduced tubulin reserve pool could lead to a reduction in new microtubule growth and disrupted maintenance of microtubule populations that need to be replaced over time. Such a disruption
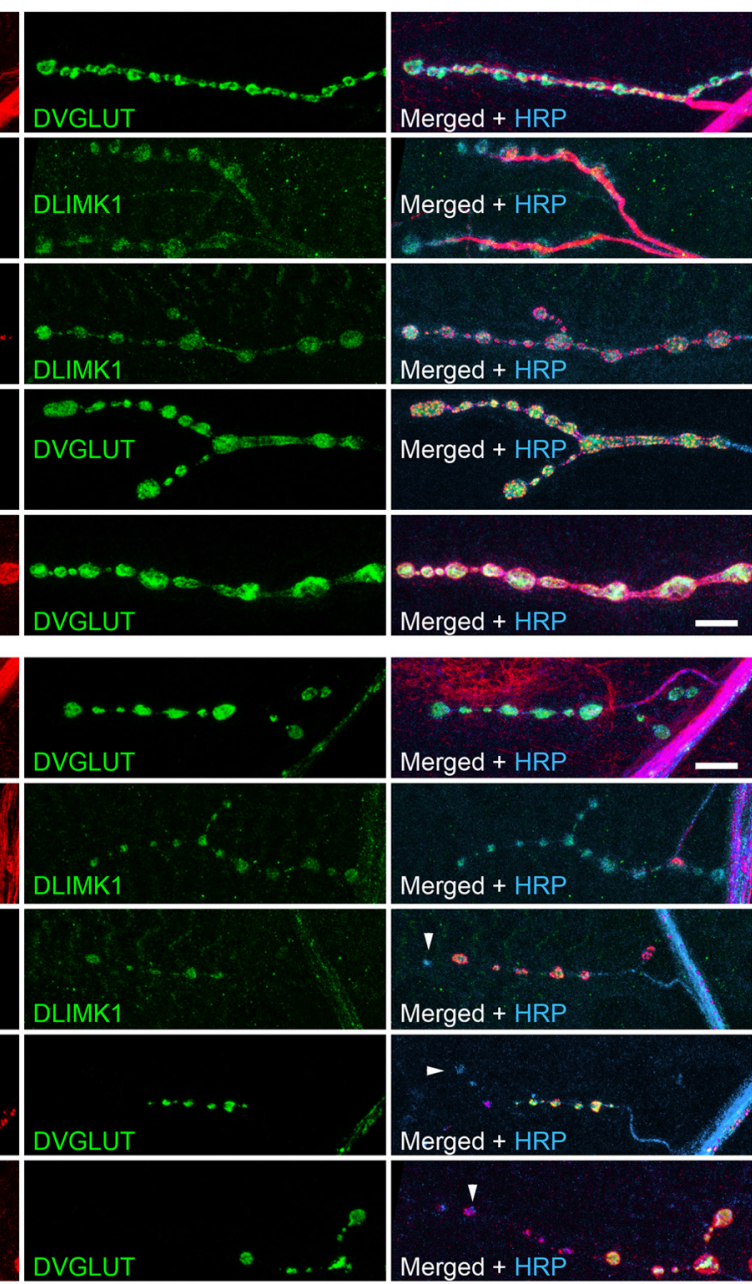

Figure 5. Molecular anatomy of axon retraction. $\boldsymbol{A}, \boldsymbol{B}$, Images of WT $(+/+)(\boldsymbol{A})$ and stathmin mutant $\left(\right.$ stai $^{B 200} /$ stathmin $\left.^{227}\right)(\boldsymbol{B})$ muscle 4 NMJs immunostained with the various antibodies shown. Arrowheads denote empty terminal patches of HRP that are separating the process into periods of preretraction, early retraction, and late retraction and the changes observed in various synaptic proteins during those periods.

to microtubule dynamics could lead directly to synaptic loss; however, previous data argue against this possibility. Loss of Futsch-stabilized synaptic microtubules is a common phenotype associated with NMJ retraction in mutations in other mutant backgrounds (Eaton et al., 2002; Pielage et al., 2005, 2008, 2011). Furthermore, futsch mutation causes a dramatic disruption in microtubule organization within the nerve terminal, but does not cause NMJ retraction (Pielage et al., 2008).

Another possibility is that Stathmin has a function within the motor axon that is necessary for normal axonal transport, a function that could be related to its microtubule-binding activity. For example, the array of phenotypes that we observe is quite similar to that observed in mutations that disrupt retrograde axonal transport (Eaton et al., 2002), including the appearance of axonal protein aggregates, impaired synapse stabilization, and altered synaptic growth. Impaired axonal transport can disrupt the retrograde axonal transport of BMP signaling from the NMJ to the motoneuron nucleus, causing synapse retraction (Eaton et al., 2002). Overexpression of the BMP ligand glass bottom boat 
(Gbb) is sufficient to suppress NMJ retraction in some mutants that disrupt axonal transport and neuromuscular stability (Massaro et al., 2009). Finally, the BMP signaling system is necessary for normal NMJ growth in Drosophila (Aberle et al., 2002; Marques et al., 2002; McCabe et al., 2003). The demonstration that synapse retractions are more severe in the posterior segments of the animal is also consistent with a primary defect in axonal transport leading to synapse destabilization. Similar anterior-toposterior gradients of phenotypic severity have been described previously in axonal transport mutants that affect synapse growth (Hurd and Saxton, 1996).

Neither neuronal expression of HA-tagged LIM kinase nor overexpression of the BMP signal Gbb in muscles (data not shown) is able to rescue synapse retraction in the stathmin mutant background. The LIM kinase functions downstream of the BMP receptor Wit to confer a portion of the synapse-stabilizing action of the BMP signaling system (Eaton and Davis, 2005). However, the other significant fraction of the synapse-stabilizing activity of BMP receptor activation is conferred by the retrograde transport of the transcription factor Mad to the motoneuron nucleus. The observation that neither neuronal LIM kinase nor postsynaptic Gbb overexpression can rescue synapse retraction in the stathmin mutant is consistent, therefore, with a defect in axonal transport in the stathmin mutant. Hence, we suggest that the impaired axonal transport of BMP signaling molecules, or other similarly essential trophic signals, may be the cause of altered synapse stability in stathmin mutants.

\section{References}

Aberle H, Haghighi AP, Fetter RD, McCabe BD, Magalhaes TR, Goodman CS (2002) wishful thinking encodes a BMP type II receptor that regulates synaptic growth in Drosophila. Neuron 33:545-558.

Banerjee S, Pillai AM, Paik R, Li J, Bhat MA (2006) Axonal ensheathment and septate junction formation in the peripheral nervous system of Drosophila. J Neurosci 26:3319-3329.

Bellen HJ, Levis RW, Liao G, He Y, Carlson JW, Tsang G, Evans-Holm M, Hiesinger PR, Schulze KL, Rubin GM, Hoskins RA, Spradling AC (2004) The BDGP gene disruption project: single transposon insertions associated with $40 \%$ of Drosophila genes. Genetics 167:761-781.

Belmont LD, Mitchison TJ (1996) Identification of a protein that interacts with tubulin dimers and increases the catastrophe rate of microtubules. Cell 84:623-631.

Borghese L, Fletcher G, Mathieu J, Atzberger A, Eades WC, Cagan RL, Rorth $\mathrm{P}$ (2006) Systematic analysis of the transcriptional switch inducing migration of border cells. Dev Cell 10:497-508.

Brand AH, Perrimon N (1993) Targeted gene expression as a means of altering cell fates and generating dominant phenotypes. Development 118:401-415.

Budnik V, Koh YH, Guan B, Hartmann B, Hough C, Woods D, Gorczyca M (1996) Regulation of synapse structure and function by the Drosophila tumor suppressor gene dlg. Neuron 17:627-640.

Collins CA, DiAntonio A (2007) Synaptic development: insights from Drosophila. Curr Opin Neurobiol 17:35-42.

Curmi PA, Andersen SS, Lachkar S, Gavet O, Karsenti E, Knossow M, Sobel A (1997) The stathmin/tubulin interaction in vitro. J Biol Chem 272:25029-25036.

Curmi PA, Gavet O, Charbaut E, Ozon S, Lachkar-Colmerauer S, Manceau V, Siavoshian S, Maucuer A, Sobel A (1999) Stathmin and its phosphoprotein family: general properties, biochemical and functional interaction with tubulin. Cell Struct Funct 24:345-357.

Daniels RW, Gelfand MV, Collins CA, DiAntonio A (2008) Visualizing glutamatergic cell bodies and synapses in the Drosophila larval and adult CNS. J Comp Neurol 508:131-152.

Eaton BA, Davis GW (2005) LIM Kinase1 controls synaptic stability downstream of the type II BMP receptor. Neuron 47:695-708.

Eaton BA, Fetter RD, Davis GW (2002) Dynactin is necessary for synapse stabilization. Neuron 34:729-741.

Featherstone DE, Rushton E, Broadie K (2002) Developmental regulation of glutamate receptor field size by nonvesicular glutamate release. Nat Neurosci 5:141-146.

Goda Y, Davis GW (2003) Mechanisms of synapse assembly and disassembly. Neuron 40:243-264.

Gonatas NK, Stieber A, Gonatas JO (2006) Fragmentation of the Golgi apparatus in neurodegenerative diseases and cell death. J Neurol Sci 246: $21-30$.

Grenningloh G, Soehrman S, Bondallaz P, Ruchti E, Cadas H (2004) Role of the microtubule destabilizing proteins SCG10 and stathmin in neuronal growth. J Neurobiol 58:60-69.

Hafezparast M, Klocke R, Ruhrberg C, Marquardt A, Ahmad-Annuar A, Bowen S, Lalli G, Witherden AS, Hummerich H, Nicholson S, Morgan PJ, Oozageer R, Priestley JV, Averill S, King VR, Ball S, Peters J, Toda T, Yamamoto A, Hiraoka Y (2003) Mutations in dynein link motor neuron degeneration to defects in retrograde transport. Science 300:808-812.

Higashi-Kovtun ME, Mosca TJ, Dickman DK, Meinertzhagen IA, Schwarz TL (2010) Importin-beta11 regulates synaptic phosphorylated mothers against decapentaplegic, and thereby influences synaptic development and function at the Drosophila neuromuscular junction. J Neurosci 30:5253-5268.

Howell B, Larsson N, Gullberg M, Cassimeris L (1999) Dissociation of the tubulin-sequestering and microtubule catastrophe-promoting activities of oncoprotein 18/stathmin. Mol Biol Cell 10:105-118.

Hummel T, Krukkert K, Roos J, Davis G, Klambt C (2000) Drosophila Futsch/22C10 is a MAP1B-like protein required for dendritic and axonal development. Neuron 26:357-370.

Hurd DD, Saxton WM (1996) Kinesin mutations cause motor neuron disease phenotypes by disrupting fast axonal transport in Drosophila. Genetics 144:1075-1085.

Jourdain L, Curmi P, Sobel A, Pantaloni D, Carlier MF (1997) Stathmin: a tubulin-sequestering protein which forms a ternary T2S complex with two tubulin molecules. Biochemistry 36:10817-10821.

Koch I, Schwarz H, Beuchle D, Goellner B, Langegger M, Aberle H (2008) Drosophila ankyrin 2 is required for synaptic stability. Neuron 58:210-222.

Lachkar S, Lebois M, Steinmetz MO, Guichet A, Lal N, Curmi PA, Sobel A, Ozon S (2010) Drosophila stathmins bind tubulin heterodimers with high and variable stoichiometries. J Biol Chem 285:11667-11680.

LaMonte BH, Wallace KE, Holloway BA, Shelly SS, Ascano J, Tokito M, Van Winkle T, Howland DS, Holzbaur EL (2002) Disruption of dynein/dynactin inhibits axonal transport in motor neurons causing late-onset progressive degeneration. Neuron 34:715-727.

Liedtke W, Leman EE, Fyffe RE, Raine CS, Schubart UK (2002) Stathmindeficient mice develop an age-dependent axonopathy of the central and peripheral nervous systems. Am J Pathol 160:469-480.

Marques G, Bao H, Haerry TE, Shimell MJ, Duchek P, Zhang B, O'Connor MB (2002) The Drosophila BMP type II receptor Wishful Thinking regulates neuromuscular synapse morphology and function. Neuron 33:529-543.

Marrus SB, Portman SL, Allen MJ, Moffat KG, DiAntonio A (2004) Differential localization of glutamate receptor subunits at the Drosophila neuromuscular junction. J Neurosci 24:1406-1415.

Massaro CM, Pielage J, Davis GW (2009) Molecular mechanisms that enhance synapse stability despite persistent disruption of the spectrin/ ankyrin/microtubule cytoskeleton. J Cell Biol 187:101-117.

McCabe BD, Marques G, Haghighi AP, Fetter RD, Crotty ML, Haerry TE, Goodman CS, O'Connor MB (2003) The BMP homolog Gbb provides a retrograde signal that regulates synaptic growth at the Drosophila neuromuscular junction. Neuron 39:241-254.

Morii H, Shiraishi-Yamaguchi Y, Mori N (2006) SCG10, a microtubule destabilizing factor, stimulates the neurite outgrowth by modulating microtubule dynamics in rat hippocampal primary cultured neurons. J Neurobiol 66:1101-1114

Osterwalder T, Yoon KS, White BH, Keshishian H (2001) A conditional tissue-specific transgene expression system using inducible GAL4. Proc Natl Acad Sci U S A 98:12596-12601.

Ozon S, Guichet A, Gavet O, Roth S, Sobel A (2002) Drosophila stathmin: a microtubule-destabilizing factor involved in nervous system formation. Mol Biol Cell 13:698-710.

Pielage J, Fetter RD, Davis GW (2005) Presynaptic spectrin is essential for synapse stabilization. Curr Biol 15:918-928.

Pielage J, Cheng L, Fetter RD, Carlton PM, Sedat JW, Davis GW (2008) A 
presynaptic giant ankyrin stabilizes the NMJ through regulation of presynaptic microtubules and transsynaptic cell adhesion. Neuron 58:195-209.

Pielage J, Bulat V, Zuchero JB, Fetter RD, Davis GW (2011) Hts/Adducin controls synaptic elaboration and elimination. Neuron 69:1039-1216.

Riederer BM, Pellier V, Antonsson B, Di Paolo G, Stimpson SA, Lutjens R, Catsicas S, Grenningloh G (1997) Regulation of microtubule dynamics by the neuronal growth-associated protein SCG10. Proc Natl Acad Sci U S A 94:741-745.

Roos J, Hummel T, Ng N, Klambt C, Davis GW (2000) Drosophila Futsch regulates synaptic microtubule organization and is necessary for synaptic growth. Neuron 26:371-382.

Saitoe M, Schwarz TL, Umbach JA, Gundersen CB, Kidokoro Y (2001) Absence of junctional glutamate receptor clusters in Drosophila mutants lacking spontaneous transmitter release. Science 293:514-517.

Schubart UK, Yu J, Amat JA, Wang Z, Hoffmann MK, Edelmann W (1996) Normal development of mice lacking metablastin (P19), a phosphoprotein implicated in cell cycle regulation. J Biol Chem 271:14062-14066.

Sepp KJ, Schulte J, Auld VJ (2001) Peripheral glia direct axon guidance across the CNS/PNS transition zone. Dev Biol 238:47-63.

Tararuk T, Ostman N, Li W, Bjorkblom B, Padzik A, Zdrojewska J, Hongisto V, Herdegen T, Konopka W, Courtney MJ, Coffey ET (2006) JNK1 phosphorylation of SCG10 determines microtubule dynamics and axodendritic length. J Cell Biol 173:265-277.

Walker RA, O’Brien ET, Pryer NK, Soboeiro MF, Voter WA, Erickson HP,
Salmon ED (1988) Dynamic instability of individual microtubules analyzed by video light microscopy: rate constants and transition frequencies. J Cell Biol 107:1437-1448.

Watabe-Uchida M, John KA, Janas JA, Newey SE, Van Aelst L (2006) The Rac activator DOCK7 regulates neuronal polarity through local phosphorylation of stathmin/Op18. Neuron 51:727-739.

Wen HL, Lin YT, Ting CH, Lin-Chao S, Li H, Hsieh-Li HM (2010) Stathmin, a microtubule-destabilizing protein, is dysregulated in spinal muscular atrophy. Hum Mol Genet 19:1766-1778.

Wernig A, Pecot-Dechavassine M, Stover H (1980) Sprouting and regression of the nerve at the frog neuromuscular junction in normal conditions and after prolonged paralysis with curare. J Neurocytol 9:278-303.

Westerlund N, Zdrojewska J, Padzik A, Komulainen E, Bjorkblom B, Rannikko E, Tararuk T, Garcia-Frigola C, Sandholm J, Nguyen L, Kallunki T, Courtney MJ, Coffey ET (2010) Phosphorylation of SCG10/stathmin-2 determines multipolar stage exit and neuronal migration rate. Nat Neurosci 14:305-313.

Yao KM, White K (1994) Neural specificity of elav expression: defining a Drosophila promoter for directing expression to the nervous system. J Neurochem 63:41-51.

Zhang YQ, Bailey AM, Matthies HJ, Renden RB, Smith MA, Speese SD, Rubin GM, Broadie K (2001) Drosophila fragile X-related gene regulates the MAP1B homolog Futsch to control synaptic structure and function. Cell 107:591-603. 